\title{
Intrinsic Prognostic Impact of Tumor-infiltrating Lymphocytes in Systemically Untreated Patients With Early-stage Triple-negative Breast Cancer
}

\author{
JI HYUN PARK ${ }^{1,2}$, HEE JIN LEE ${ }^{3}$, SAE BYUL LEE ${ }^{4}$, JIN-HEE AHN ${ }^{2}$, JEONG EUN KIM ${ }^{2}$, \\ KYUNG HAE JUNG ${ }^{2}$, GYUNGYUB GONG ${ }^{3}$, BYUNG-HO SON ${ }^{4}$, SEI-HYUN AHN ${ }^{4}$ and SUNG-BAE KIM ${ }^{2}$ \\ ${ }^{1}$ Department of Hemato-Oncology, Konkuk University Medical Center, \\ Univeristy of Konkuk College of Medicine, Seoul, Republic of Korea; \\ Departments of ${ }^{2}$ Oncology, ${ }^{3}$ Pathology, ${ }^{4}$ Surgery, Asan Medical Center, \\ University of Ulsan College of Medicine, Seoul, Republic of Korea
}

\begin{abstract}
Background: The prognostic value of tumorinfiltrating lymphocytes (TILs) in curatively resected, but systemically untreated early-stage triple-negative breast cancer (TNBC) was investigated. Materials and Methods: Patients with systemically untreated early TNBC between 1999 and 2012 were retrospectively reviewed. A low TIL level was defined as the presence of $\leq 10 \%$ stromal TILs Relapses were classified into locoregional and distant relapse. The primary endpoint was breast cancer-specific survival (BCSS). Results: In 72 patients, the median TIL value was 10\%, and low TIL status was found in 54.2\%. Patients with $p T 1$ and nodal-positive disease constituted $75 \%$ and $11.1 \%$, respectively. With a median followup of 99 months, $26.4 \%$ patients experienced relapse; local in $63.2 \%$, distant in $36.8 \%$, and $9.7 \%$ died of disease progression. A low TIL level was significantly associated with distant relapse $(p=0.013)$, and inferior 10-year BCSS, which was consistently observed in patients with Tla/b or NO disease. Conclusion: A low TIL level seems to be an intrinsic prognostic factor in systemically untreated patients with early-stage TNBC, even in the Tla/bNO subset.
\end{abstract}

Tumor infiltrating lymphocytes (TIL) are regarded as key immune-related players in triple-negative breast cancer (TNBC), a special subtype of breast cancer harboring intrinsic heterogeneity (1-5). As TILs vigorously communicate with

This article is freely accessible online.

Correspondence to: Sung-Bae Kim, MD, Ph.D., Department of Oncology, Asan Medical Center, University of Ulsan College of Medicine, 88 Olympic-ro 43-gil, Songpa-gu, Seoul 05505, Korea. Tel.: +82 230103217, Fax: +82 230108772, e-mail: sbkim3@amc.seoul.kr

Key Words: Tumor-infiltrating lymphocyte, TIL, triple-negative breast cancer, TNBC, systemically untreated, prognosis. the tumor microenvironment, they reflect host antitumor immunity engaged with adaptive immune activation $(6,7)$. As an abundance of TILs in TNBC may contribute to sculpturing a favorable TME, prohibiting tumor progression, its prognostic impact has been recently highlighted in patients with early-stage TNBC (8-17). Although a richness of TILs strongly suggested better treatment response and survival outcomes in the context of systemic treatment, both in neoadjuvant and adjuvant settings (9-11, 13, 18-20), there is a relative lack of data investigating its prognostic role in the systemically untreated subset of patients with early-stage TNBC. Under current standardized treatment guidelines, most patients with early TNBC uniformly receive intensive neoadjuvant or adjuvant treatment with concerns about relatively more detrimental clinical outcomes of advanced TNBC. However, considering its heterogeneous biological nature, there is an unmet need for further personalized treatment for patients with early TNBC on the basis of their molecular signatures incorporating TILs.

Thus, we attempted to evaluate the prognostic value of TILs in association with the pattern of relapse and long-term breast cancer-specific survival (BCSS) in Asian patients curatively resected for early-stage TNBC, exclusively in the absence of adjuvant chemotherapy. It might give us a cue to define the intrinsic role of TILs as a prognostic biomarker in early TNBC. In addition, in order to further clarify the relevance of TILs in these treatment-naive patients, we focused on its significance in clinically more quiescent subsets with small primary $(\leq 1 \mathrm{~cm})$ or pathologically node-negative disease.

\section{Materials and Methods}

Patients. We retrospectively collected and reviewed medical records of patients with early TNBC who did not receive any systemic adjuvant treatment after curative resection between January 1999 to December 2012 at Asan Medical Center (Seoul, Korea). Patients with pathologically proven TNBC were eligible regardless of their 
stage and histological subtypes. We enrolled patients who received adjuvant radiotherapy alone without systemic chemotherapy. However, patients with resected metastatic disease before surgery, bilateral or inflammatory breast cancer, or who received any neoadjuvant treatment were excluded. Any history of past or concurrent malignancies was not allowed except for those who had fully recovered from non-melanoma skin cancer or cervical carcinoma in situ.

Relapses were classified into locoregional relapse (LRR), and relapses with distant metastasis (DR). LRR was defined as disease relapse clinically or pathologically confirmed in the ipsilateral breast or chest wall, or ipsilateral axillary, supraclavicular, infraclavicular or internal mammary lymph nodes.

The study protocol was reviewed and approved by the Institutional Review Board (IRB approval number: 2014-1061) of Asan Medical Center and was conducted in full accordance with the guidelines for Good Clinical Practice and the Declaration of Helsinki.

Histological evaluation and immunohistochemistry. TNBC was immunohistochemically defined as estrogen receptor (ER), progesterone receptor (PR), and human epidermal growth factor receptor 2 (HER2)/neu were all negative (0 or $1+$ by Immunohistochemistry). ER and PR positivity were defined as strong nuclear staining in at least $3 / 8$ of the tumor cells examined. HER2/neu positivity was defined as strong (3+) membranous staining in at least $10 \%$ of tumor cells, whereas scores of 0 to $2+$ were regarded as negative. However, primary tumor negative for ER, PR, and HER2/neu 2+ by IHC without fluorescent in situ hybridization results was not classified as TNBC.

For pathologically confirmed TNBC tumors, entire tumor beds from surgery were stained with hematoxylin and eosin, and analyzed to determine histological and nuclear grades, overall (including in situ carcinoma) and invasive cancer size and cellularity, $\mathrm{pT} / \mathrm{pN}$ stage, Ki-67 labeling index and TILs. TILs were defined as the mean percentage of stromal area of invasive carcinoma infiltrated by lymphocytes and plasma cells in $10 \%$ increments, which followed the TIL Working Group recommendations (21), and was evaluated by two breast pathologists (HJL, GG). In the study, cases were defined as having a low level of TILs when stromal TILs $\leq 10 \%$, and a high level when stromal TILs $>10 \%$ for analysis. As proposed by Denkert et $a l$., we also categorized lymphocyte-predominant breast cancer (LPBC) as that involving $\geq 50 \%$ lymphocytic infiltration of either tumor stroma or cell nests (22). Histological subtype of TNBC was defined by 2012 World Health Organization (WHO) criteria, and grade by the modified Bloom-Richardson classification (23).

Statistical analysis. The primary endpoint was to evaluate prognostic significance of TILs for BCSS, defined as the time interval between the date of diagnosis to the date of death due to breast cancer. Relapse-free survival (RFS) was evaluated as a secondary endpoint, which was defined as the time interval between the date of diagnosis to the date of first objective detection of breast cancer relapse regardless of its type. Pearson's chi-square, Fisher's exact tests, and Kaplan-Meier method with log-rank test were used in univariate analyses. Chi-square and Fisher's exact tests were applied for comparison of categorical variables, and Kaplan-Meier method using the log-rank test was used for survival estimation. Significant covariates in univariate analysis were applied to a multivariate analysis using Cox proportional hazards model to determine the hazard ratios (HRs) and 95\% confidence intervals (CI). All $p$-values were two-sided, and a value of $p<0.05$ was considered statistically significant. PASW Statistics (version 20; IBM Co., Armonk, NY, USA) was used for all statistical analysis.

\section{Results}

Patient characteristics. A total of 72 patients with evaluable TILs were finally analyzed, and their clinicopathological characteristics are summarized in Table I. The median age was 57 years (range $=24$ to 81 years). Pathological stage I was most frequently observed $(69.4 \%)$. The median size of primary breast lesions was $1.5 \mathrm{~cm}$, and pathological nodal involvement was found in eight $(11.1 \%)$ patients. Invasive ductal carcinoma (IDC) constituted the most frequent histopathological subtype (83.3\%). A high Ki-67 $(\geq 0 \%)$ index was observed in $48.6 \%$ of patients. Adjuvant radiotherapy was performed in 29 patients $(37.2 \%)$. The reason for no adjuvant chemotherapy were mostly refusal by patients or guardians regardless of physician's recommendation $(\mathrm{n}=44,61.1 \%)$, followed by cases unrecommendable due to favorable histological subtype or small size of IDC without nodal involvement $(n=26$, $36.1 \%)$. There were three $(4.2 \%)$ patients with a family history of breast cancer; one had a daughter with breast cancer, and others had sisters with breast cancer, but none had breast cancer gene (BRCA1/2) aberrations.

TILs and correlation with relapse patterns. The median TIL value was $10 \%$ (range $=1-90 \%$ ) for the cohort overall. LPBC ( $\geq 50 \%$ TILs) was observed in $27.8 \%$ (20 out of 78 patients), whereas 39 patients $(54.2 \%)$ demonstrated a low TIL level. Patients with a low TIL level significantly more frequently had IDCs rather than other histological subtypes. Distribution of TILs was not associated with pathological nodal status or size of primary tumor in the cohort, but the TIL level was significantly correlated with the pattern of relapse (Table I).

In a total of $19(26.4 \%)$ relapsed patients, LRRs were found in 12 patients $(63.2 \%)$, whereas relapses accompanying distant metastasis (DR) were observed in seven patients $(36.8 \%)$, including four who harbored both locoregional and distant metastatic lesions (5.6\%). Of LRRs, local relapse alone was observed in $58.3 \%$ of patients $(n=7)$, presenting as isolated breast or ipsilateral skin or chest wall lesions after mastectomy, whereas regional relapses with ipsilateral lymph node metastases were found in five patients $(41.7 \%)$. The lung was the most frequently involved distant metastatic organ, with six $(85.7 \%)$ out of seven patients with DR presenting multiple lung metastases (Table II). 
Table I. Patient characteristics, and comparison according to level of tumor-infiltrating lymphocytes (TILs).

\begin{tabular}{|c|c|c|c|c|c|}
\hline & & Overall & Low TILs $(\leq 10 \%)$ & High TILs $(>10 \%)$ & $p$-Value \\
\hline Total patients & & $72(100.0)$ & $39(54.2)$ & $33(45.8)$ & \\
\hline Age, years & Median (range) & $57.0(24-81)$ & $58.0(31-80)$ & $53(24-81)$ & 0.553 \\
\hline Gender, n (\%) & Female & $72(100.0)$ & $39(54.2)$ & $33(45.8)$ & N/A \\
\hline \multirow[t]{2}{*}{ Type of surgery, n (\%) } & $\mathrm{BCO}$ & $49(68.1)$ & $24(61.5)$ & $25(75.8)$ & 0.217 \\
\hline & Mastectomy & $23(31.9)$ & $15(38.5)$ & $8(24.2)$ & \\
\hline \multirow[t]{3}{*}{ Pathological TNM stage, n (\%) } & I & $50(69.4)$ & $26(66.7)$ & $24(72.7)$ & 0.539 \\
\hline & II & $19(26.4)$ & $12(30.8)$ & $7(21.2)$ & \\
\hline & III & $3(4.2)$ & $1(2.6)$ & $2(6.1)$ & \\
\hline \multirow{8}{*}{ Primary tumor size, $\mathrm{cm}$} & Median (range) & $1.5(0.2-10.0)$ & $1.5(0.3-10.0)$ & $1.2(0.2-5.5)$ & 0.201 \\
\hline & pT1 & $54(75.0)$ & $27(69.2)$ & $27(81.8)$ & 0.574 \\
\hline & $1 \mathrm{a}$ & $18(33.3)$ & $8(29.6)$ & $10(37.0)$ & \\
\hline & $1 \mathrm{~b}$ & $11(20.4)$ & $5(18.5)$ & $6(22.2)$ & \\
\hline & $1 \mathrm{c}$ & $25(46.3)$ & $14(51.9)$ & $11(40.7)$ & \\
\hline & pT2 & $14(17.9)$ & $9(23.1)$ & $5(15.2)$ & \\
\hline & pT3 & $3(3.8)$ & $2(5.1)$ & $1(3.0)$ & \\
\hline & pT4 & $1(1.3)$ & $1(2.6)$ & $0(0.0)$ & \\
\hline \multirow[t]{3}{*}{ Pathologic nodal involvement, $\mathrm{n}(\%)$} & Overall & $8(11.1)$ & $3(7.7)$ & $5(15.2)$ & \\
\hline & $\mathrm{pN} 1$ & $6(8.3)$ & $3(7.7)$ & $3(9.1)$ & \\
\hline & $\mathrm{pN} 2$ & $2(2.8)$ & $0(0.0)$ & $2(6.1)$ & \\
\hline \multirow[t]{6}{*}{ Histological subtype, n (\%) } & Invasive ductal carcinoma (IDC) & $60(83.3)$ & $27(69.2)$ & $33(100)$ & 0.032 \\
\hline & Metaplastic & $5(6.9)$ & $5(12.8)$ & $0(0)$ & \\
\hline & Adenoid cystic & $4(5.6)$ & $4(10.3)$ & $0(0)$ & \\
\hline & Invasive papillary & $1(1.4)$ & $1(2.6)$ & $0(0)$ & \\
\hline & Invasive apocrine & $1(1.4)$ & $1(2.6)$ & $0(0)$ & \\
\hline & Secretory & $1(1.4)$ & $1(2.6)$ & $0(0)$ & \\
\hline \multirow[t]{2}{*}{ Histological differentiation, $\mathrm{n}(\%)$} & Well or moderate & $28(39.4)$ & $17(44.7)$ & $11(33.3)$ & 0.317 \\
\hline & Poor & $43(60.6)$ & $21(55.3)$ & $22(66.7)$ & \\
\hline \multirow[t]{3}{*}{ Ki-67 index, \% } & Median (range) & $10(0-80)$ & $10(0-80)$ & $20(0-80)$ & 0.445 \\
\hline & High $(\geq 20 \%)$ & $35(48.6)$ & $18(46.2)$ & $17(51.5)$ & 0.813 \\
\hline & Low $(<20 \%)$ & $37(51.4)$ & $21(53.8)$ & $16(48.5)$ & \\
\hline TILs, \% & Median (range) & $10(1-90)$ & $2(1-10)$ & $33(20-90)$ & $<0.001$ \\
\hline Adjuvant radiotherapy, n (\%) & Received & $29(37.2)$ & $16(41.0)$ & $13(39.4)$ & 0.888 \\
\hline \multirow{3}{*}{$\begin{array}{l}\text { Reason for withholding adjuvant } \\
\text { chemotherapy, n (\%) }\end{array}$} & Patient/guardian refusal & $44(61.1)$ & $27(69.2)$ & $17(51.5)$ & 0.138 \\
\hline & Physician's discretion & $26(36.1)$ & $12(30.8)$ & $14(42.4)$ & \\
\hline & Special medical condition ${ }^{\dagger}$ & $2(2.8)$ & $0(0.0)$ & $2(6.1)$ & \\
\hline
\end{tabular}

BCO: Breast-conserving operation; N/A: not applicable; TILs: tumor-infiltrating lymphocytes. ${ }^{\dagger}$ Autoimmune hepatitis, pregnancy.

Of note, a low TIL level was significantly associated with DR (low vs. high level: $58.3 \%$ vs. $0.0 \%, p=0.013$ ), although there was no relationship between overall relapse and TIL level (Table II). However, other clinicopathological variables including pathologic nodal involvement or tumor primary size did not significantly affect the tendency for DR (Table III).

Prognostic significance of TILs. With a median follow-up period of 99 months (95\% CI=91-107 months) for survivors, $19(26.4 \%)$ patients experienced relapse, and seven patients $(9.7 \%)$ died of breast cancer progression, which made 10 year BCSS and RFS rates of $90.8 \%$ and $63.1 \%$, respectively.

Univariate and multivariate analyses for TILs. In a univariate analysis for BCSS, a low TIL level and relapse with distant metastasis were significantly associated with inferior 10-year BCSS rates $(p=0.009$ and $p<0.001$, respectively) (Figure 1, Table IV). Patients with relapse had a significantly inferior 10-year BCSS rate, but LRR alone did not significantly affect BCSS $(p=0.208)$. Patients with pathological nodal metastases and primary tumor size $>1 \mathrm{~cm}$ had an inferior 10-year BCSS rates despite not reaching statistical significance $(93.2 \%$ vs. $75.0 \%, p=0.172$; and $95.5 \%$ vs. $87.8 \%, p=0.183)$. Adjuvant radiotherapy was not correlated with long-term survival outcomes. Other pathological parameters including Ki-67 proliferative index and histological grade did not significantly affect the 10-year BCSS rate. Interestingly, histological subtype also did not influence breast cancer-specific death in that patients with IDC did not have inferior survival outcomes compared with those with non-IDC subtypes (Table IV). 
Table II. Patterns of relapse and their association with the level of tumor-infiltrating lymphocytes (TILs).

\begin{tabular}{|c|c|c|c|c|c|}
\hline & & \multicolumn{4}{|c|}{ Patients, n (\%) } \\
\hline & & $\begin{array}{l}\text { Overall } \\
(\mathrm{N}=72)\end{array}$ & $\begin{array}{c}\text { Low TILs }(\leq 10 \%) \\
(\mathrm{N}=39,54.2 \%)\end{array}$ & $\begin{array}{l}\text { High TILs }(>10 \%) \\
(\mathrm{N}=33,45.8 \%)\end{array}$ & $p$-Value \\
\hline Relapse & Any type & $19(26.4)$ & $12(30.8)^{\dagger}$ & $7(21.2)^{\dagger}$ & 0.428 \\
\hline \multirow{5}{*}{ Locoregional only } & Overall & $12(63.2)$ & $5(41.7)$ & $7(100.0)$ & 0.013 \\
\hline & Local & $7(58.3)$ & $3(60.0)$ & $4(57.1)$ & \\
\hline & Isolated breast & $5(71.5)$ & $2(40.0)$ & $3(42.9)$ & \\
\hline & Ipsilateral skin/chest wall & $2(28.6)$ & $1(20.0)$ & $1(14.3)$ & \\
\hline & Regional lymph nodes & $5(41.7)$ & $2(40.0)$ & $3(42.9)$ & \\
\hline Distant & Overall & $7(36.8)$ & $7(58.3)$ & $0(0.0)$ & \\
\hline \multirow[t]{5}{*}{ Metastatic site } & Lung & & $6(85.7)$ & & \\
\hline & Distant lymph nodes & & $2(28.6)$ & & \\
\hline & Bone & & $2(28.6)$ & & \\
\hline & Liver & & $1(14.3)$ & & \\
\hline & Brain & & $1(14.3)$ & & \\
\hline
\end{tabular}

Among 19 patients with relapse: $\uparrow 63.2 \%$ and $\uparrow \uparrow 36.8 \%$.

In an exploratory multivariate analysis, we applied significant covariates from the univariate analysis, but used a low TIL level instead of DR on the basis of their significant statistical association. We additionally incorporated primary tumor size larger than $1 \mathrm{~cm}$ and pathological nodal involvement on the basis of current guidelines for adjuvant chemotherapy in early TNBC. The result revealed a low TIL level as one final prognostic index for BCSS, although it did not reach the statistical significance threshold $(p=0.180$, data not shown).

Role of TILs in patients with NO or T1a/b TNBC. Noticeably, in the pathologically node-negative TNBC subset $(\mathrm{n}=64$, $88.9 \%$ ), only a low TIL level led to a significantly inferior 10 -year BCSS rate $(88.3 \%$ vs. $100 \%, p=0.036)$, which was consistent in patients with small primary tumors $(\leq 1 \mathrm{~cm})$. In 28 patients with both small primary and node-negative TNBC, the 10-year BCSS rate was lower in those patients with a low TIL level (90.9\% vs. 100.0\%, $p=0.340)$. More importantly, two relapses observed in the subset were in patients with a low TIL level, which demonstrated a relevant association between a low TIL level and inferior 10-year RFS (68.2\% vs. $100.0 \%, p=0.072)$. Of these two patients, one with $10 \%$ TILs and $0.3 \mathrm{~cm}$ primary tumor surprisingly demonstrated DR including multiple lung metastases and eventually died of disease progression (Figure 2).

\section{Discussion}

The present study highlights the natural prognostic significance of TILs in patients with systemically untreated early-stage TNBC after curative resection, demonstrating a significant association of a low TIL level with a tendency for
DR and poor long-term survival outcome. Moreover, a low TIL level even predicted the patients with relatively unfavorable BCSS in more quiescent TNBC subsets with node-negative or small primary tumors, which is currently not under consideration in adjuvant chemotherapy.

Although the prognostic value of TILs in early-stage TNBC was extrapolated from several randomized adjuvant trials and meta-analyses $(9,11,13,24)$, few have evaluated its prognostic significance in systemically untreated patients. However, two recent studies added evidence on the prognostic significance of TILs in the absence of systemic chemotherapy. Dieci et al. made an initial successful attempt to elucidate the prognostic impact of TILs in patients with systemically untreated early breast cancer, and suggested an inexplicit but interesting possibility of withholding adjuvant chemotherapy for those with early TNBC or HER2-positive BC with abundant TILs (10). Most recently, Leon-Ferre et $a l$. also focused on the prognostic impact of TILs in early TNBC, including patients who never received adjuvant chemotherapy, and demonstrated a prognostic significance of TILs in the whole cohort (12). What was more surprising, however, was the independently maintained prognostic value of TILs in systemically untreated patients, even for the T1N0 subset. These findings might be possibly explained by the intrinsic immunogenic role of TILs in TNBC, as they harness the endogenous antitumor immune reaction and induce favorable TME modification whether chemotherapy is applied or not $(2,4,12,25)$.

As a low TIL level exclusively predicted poor 10 -year BCSS and higher risk of DR in our study, we speculated the TIL level to be a critical determinant of long-term survival in these patients. Furthermore, we made an additional suggestion of the prognostic value of a low TIL 
Table III. Univariate analysis for risk factors of distant relapse with clinicopathological parameters.

\begin{tabular}{|c|c|c|c|c|c|}
\hline & & \multicolumn{4}{|c|}{ Distant relapse, n (\%) } \\
\hline & & Total, n (\%) & Yes & No & $p$-Value \\
\hline Total patients & & $72(100.0)$ & $7(9.7)$ & $65(90.3)$ & \\
\hline Age & $\geq 60$ Years & $34(47.2)$ & $4(57.1)$ & $34(52.3)$ & 0.563 \\
\hline \multirow[t]{2}{*}{ Type of surgery } & $\mathrm{BCO}$ & $49(68.1)$ & $4(57.1)$ & $45(69.2)$ & 0.673 \\
\hline & Mastectomy & $23(31.9)$ & $3(42.9)$ & $20(30.8)$ & \\
\hline \multirow[t]{3}{*}{ Pathological TNM stage } & I & $50(69.4)$ & $4(57.1)$ & $46(70.8)$ & 0.525 \\
\hline & II & $19(26.4)$ & $3(42.9)$ & $16(24.6)$ & \\
\hline & III & $3(4.2)$ & $0(0.0)$ & $3(4.6)$ & \\
\hline Primary tumor size & $>1 \mathrm{~cm}$ & $43(59.7)$ & $6(85.7)$ & $37(56.9)$ & 0.230 \\
\hline Pathological nodal status & Positive & $8(11.1)$ & $1(14.3)$ & $7(10.8)$ & 0.578 \\
\hline \multirow[t]{6}{*}{ Histological subtype } & IDC & $60(83.3)$ & $5(71.4)$ & $55(84.6)$ & 0.832 \\
\hline & Metaplastic & $5(6.9)$ & $1(14.3)$ & $4(6.2)$ & \\
\hline & Adenoid cystic & $4(5.6)$ & $1(14.3)$ & $3(4.6)$ & \\
\hline & Invasive papillary & $1(1.4)$ & $0(0.0)$ & $1(1.5)$ & \\
\hline & Invasive apocrine & $1(1.4)$ & $0(0.0)$ & $1(1.5)$ & \\
\hline & Secretory & $1(1.4)$ & $0(0.0)$ & $1(1.5)$ & \\
\hline Histological grade & Poorly differentiated & $43(60.6)$ & $4(66.7)$ & $39(60.0)$ & $>0.99$ \\
\hline TILs & $\leq 10 \%$ & $39(54.2)$ & $7(100)$ & $32(49.2)$ & 0.013 \\
\hline Ki-67 index & $\geq 20 \%$ & $35(48.6)$ & $3(42.9)$ & $34(49.2)$ & $>0.99$ \\
\hline Lymphovascular invasion & Yes & $5(6.9)$ & $1(14.3)$ & $4(6.2)$ & 0.410 \\
\hline Adjuvant radiotherapy alone & Yes & $29(37.2)$ & $2(28.6)$ & $27(41.5)$ & 0.694 \\
\hline
\end{tabular}

BCO: Breast-conserving operation; IDC: invasive ductal carcinoma; TILs: tumor-infiltrating lymphocytes.

Table IV. Univariate analysis for 10-year breast cancer-specific survival (BCSS) rate.

\begin{tabular}{|c|c|c|c|}
\hline \multicolumn{2}{|l|}{ Clinicopathological variable } & \multirow{2}{*}{$\frac{\text { 10-Year BCSS rate (\%) }}{94.1 \text { vs. } 87.2}$} & \multirow{2}{*}{$\frac{p \text {-Value }}{0.437}$} \\
\hline Old age & $\geq 60$ vs. $<60$ Years & & \\
\hline Type of surgery & BCO vs. mastectomy & $93.1 \mathrm{vs} .86 .5$ & 0.216 \\
\hline Pathological TNM stage & I $v s$. II $v s$. III & $93.2 v s .83 .9$ vs. 100.0 & 0.663 \\
\hline Primary tumor size & $>1$ vs. $\leq 1 \mathrm{~cm}$ & 95.5 vs. 87.8 & 0.183 \\
\hline Pathological nodal status & Positive $v s$. negative & 93.2 vs. 75.0 & 0.172 \\
\hline Histological subtypes & IDC vs. other & 90.7 vs. 91.7 & 0.405 \\
\hline Histological grade & Poorly differentiated $v s$. other & 92.0 vs. 89.9 & 0.702 \\
\hline TILs & $\leq 10 \%$ vs. $>10 \%$ & 100.0 vs. 83.4 & 0.009 \\
\hline High Ki-67 index & $\geq 20 \%$ vs. $<20 \%$ & $91.6 v s .91 .4$ & 0.766 \\
\hline Lymphovascular invasion & Yes $v s$. no & 91.6 vs. 80.0 & 0.524 \\
\hline IHC HER2 expression & Positive $v s$. negative & 94.5 vs. 77.0 & 0.152 \\
\hline Adjuvant radiotherapy alone & Yes $v s$. no & 88.0 vs. 94.7 & 0.224 \\
\hline Any type of relapse & Yes vs. no & 100.0 vs. 67.7 & $<0.001$ \\
\hline Locoregional relapse alone & Yes vs. no & 92.8 vs. 82.5 & 0.519 \\
\hline Relapse with distant metastasis & Yes vs. no & 96.4 vs. 42.9 & $<0.001$ \\
\hline
\end{tabular}

BCO: Breast-conserving operation; IDC: invasive ductal carcinoma; TILs: tumor-infiltrating lymphocytes; IHC: immunohistochemical; HER2: human epidermal receptor 2 .

level in clinically more quiescent subsets with nodenegative or small primary tumors (T1a/b or N0). Given that adjuvant chemotherapy could be optionally spared in these patients with the concept of a de-escalating strategy, it warns us of the possible underestimation of relapse or risk of death in these subsets, showing the necessity for further refinement of adjuvant treatment strategy. PathologicaI nodal involvement was not significantly associated with DR or TILs in this cohort, which was discordant from a previous study that suggested a close relationship between nodal status and TILs (9). However, although statistical significance was not met, patients with pathological nodal 

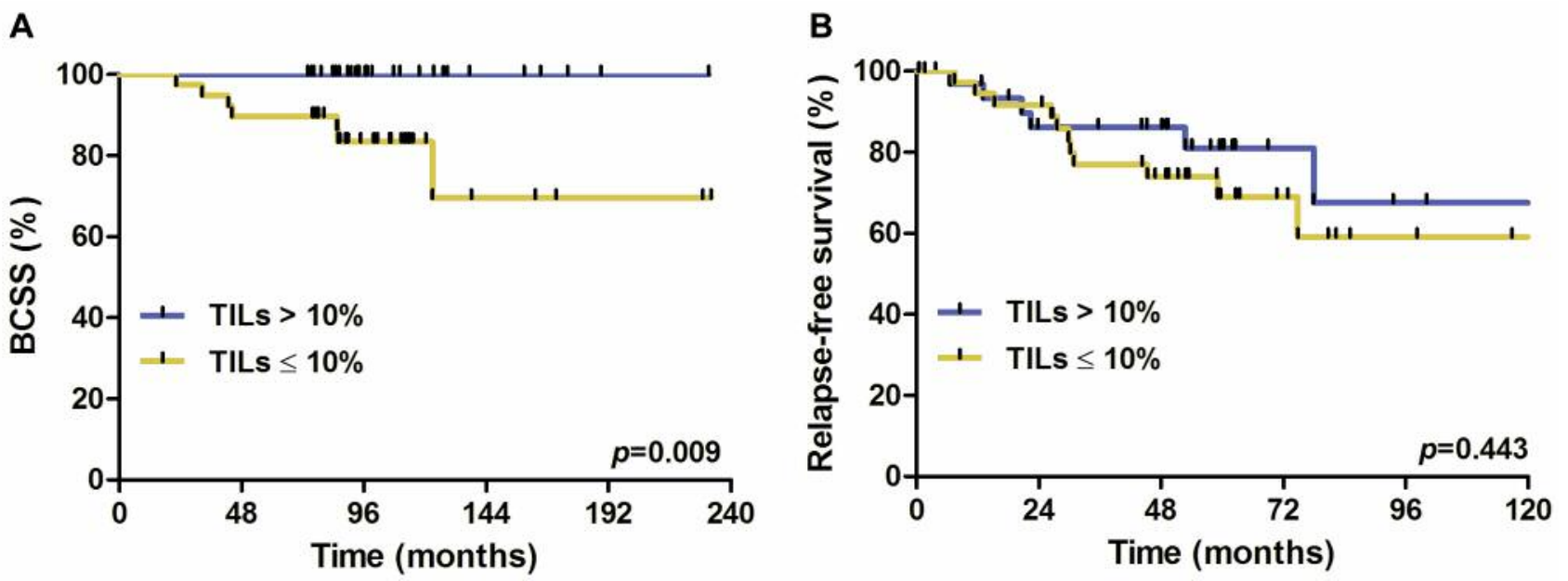

Figure 1. Breast cancer-specific (BCSS) (A) and relapse-free (B) survival in the whole patient cohort (N=78) according to the level of tumorinfiltrating lymphocytes (TILs).

metastases demonstrated relatively unfavorable long-term BCSS as reported in previous studies $(9,10,12)$, which should be further investigated in a larger prospective cohort.

Our study has several methodological limitations. Firstly, it inevitably has a limitation from its retrospective nature and small sample sized from a single center. Secondly, it was also unable to avoid selection bias because systemically untreated patients might be extracted from the entire cohort owing to certain clinicopathological features. For example, our landmark study revealed that systemically untreated patients were significantly older (median age 64 years) with more node-negative (N0: 82\%) and smaller tumors (T1: 68\%) compared to patients who received adjuvant chemotherapies (12). In comparison, the cohort of the present study comprised relatively younger patients (median age 57 years), and those with slightly more nodenegative $(88.9 \%)$ or small primary tumors (T1: $75.0 \%$ ). Thus, the present study might incompletely reflect the general population with early TNBC but rather focused on the subset with more favorable biological features. However, particularly in terms of TIL, the median percentage of TILs and proportion of those with LPBC were comparable $(9,10,12)$. Lastly, we incorporated all heterogeneous histological subtypes of TNBC other than IDC which included some with relatively favorable biological nature such as adenoid cystic carcinoma. However, we observed no significant difference in longterm BCSS according to histological subtype. Moreover, we observed DR and eventual death in one patient $(25 \%)$ with a low TIL level among four with adenoid cystic carcinomas, which suggested a more powerful prognostic impact of TILs beyond histological biology. Despite these limitations, our study seems to be the first evaluation in an Asian population with a long-term follow-up, which addressed the prognostic value of TILs exclusively in systemically untreated patients with early TNBC.

In conclusion, we suggest a low TIL level $(\leq 10 \%)$ to be an independent and intrinsic prognostic factor in systemically untreated patients with early-stage TNBC, which strongly predicts the risks of DR and poor long-term BCSS. Therefore, patients with a low TIL level, even in the T1a/bN0 subset, may be potential candidates for adjuvant chemotherapy regardless of nodal status or primary tumor size. In order to establish a personalized adjuvant treatment strategy in early-stage TNBC, TILs should be incorporated as another determining factor of chemotherapy, and thus be routinely evaluated.

\section{Conflicts of Interest}

The Authors have no financial disclosures related to this study.

\section{Authors' Contributions}

JHP reviewed the literature, prepared and designed the analysis of data, and drafted and revised the article. HJL, our pathologist and an expert for TILs in breast cancer, made key contributions to the evaluation and interpretation of TILs. GG and HJL made a thorough pathological review and also revised histological methodology in the article. SBL gave a core assistance in initial data collection and developing a raw registry, which was fundamentally supported by our surgeons BHS and SHA. JK, JHA, and $\mathrm{KHJ}$ equally contributed to enrollment of patients as expert medical oncologists of breast cancer. SBK initially inspired the conception and design of the study, and finally gave final approval of the version to be published. All Authors read and approved the final article. 

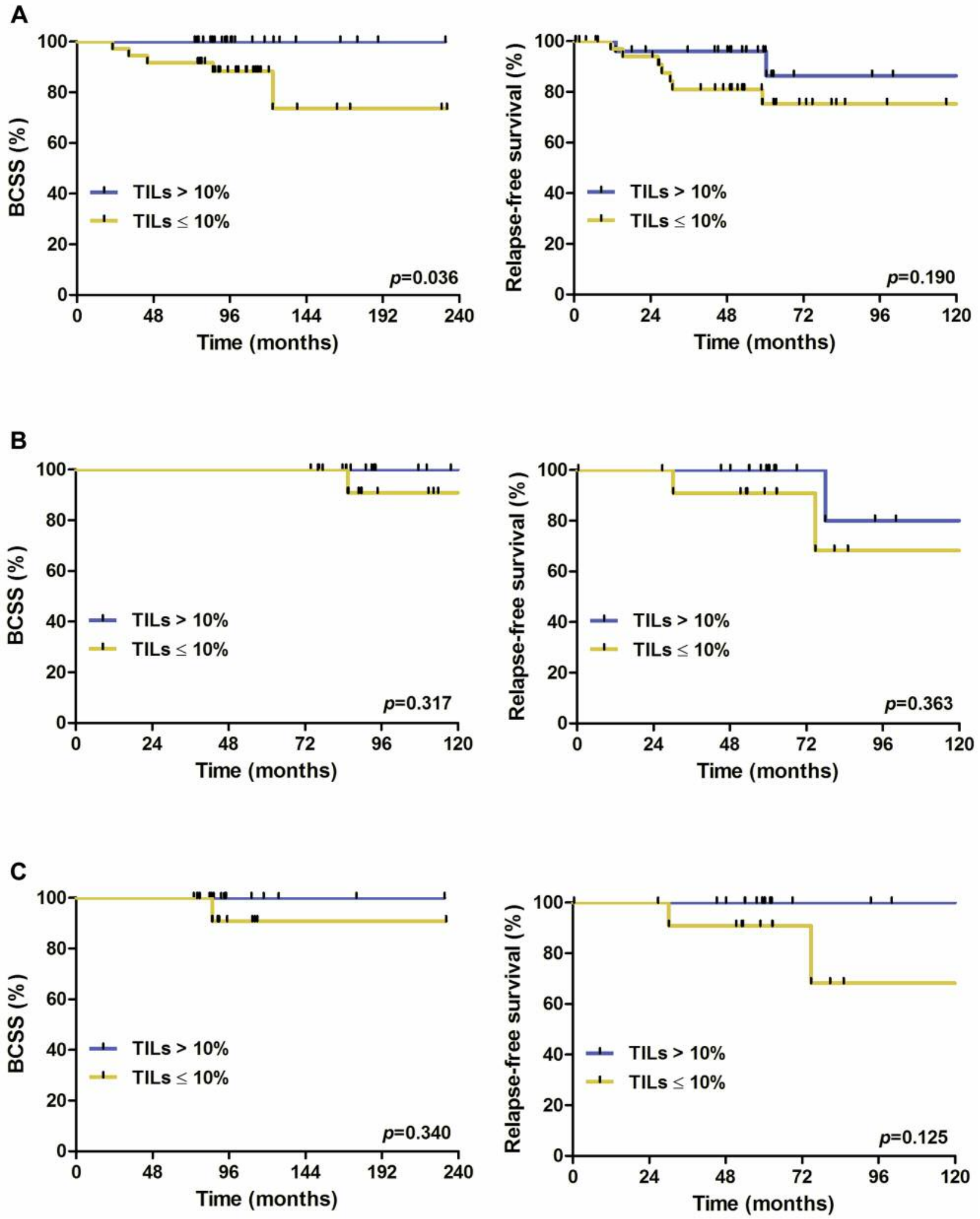

Figure 2. Breast cancer-specific (BCSS; left panel) and relapse-free survival (right panel) outcomes in patients with node-negative tumor (N=64) $(A)$, small primary $(\leq 1 \mathrm{~cm})$ tumor $(N=29)(B)$, and those with both node-negative and small primary tumors $(N=28)(C)$ according to the level of tumor-infiltrating lymphocytes (TILs). 


\section{References}

1 Liu F, Lang R, Zhao J, Zhang X, Pringle GA, Fan Y, Yin D, Gu F, Yao Z and Fu L: CD8(+) cytotoxic T-cell and FOXP3(+) regulatory $\mathrm{T}$-cell infiltration in relation to breast cancer survival and molecular subtypes. Breast Cancer Res Treat 130(2): 645-655, 2011. PMID: 21717105. DOI: 10.1007/ s 10549-011-1647-3

2 Bianchini G, Balko JM, Mayer IA, Sanders ME and Gianni L: Triple-negative breast cancer: Challenges and opportunities of a heterogeneous disease. Nat Rev Clin Oncol 13(11): 674-690, 2016. PMID: 27184417. DOI: $10.1038 /$ nrclinonc 2016.66

3 Denkert C, Liedtke C, Tutt A and von Minckwitz G: Molecular alterations in triple-negative breast cancer-the road to new treatment strategies. Lancet 389(10087): 2430-2442, 2017. PMID: 27939063. DOI: 10.1016/S0140-6736(16)32454-0

4 Park JH, Ahn JH and Kim SB: How shall we treat early triplenegative breast cancer (TNBC)? From the current standard to upcoming immuno-molecular strategies. ESMO Open 3(Suppl 1): e000357, 2018. PMID: 29765774. DOI: 10.1136/esmoopen2018-000357

5 Burstein MD, Tsimelzon A, Poage GM, Covington KR, Contreras A, Fuqua SA, Savage MI, Osborne CK, Hilsenbeck SG, Chang JC, Mills GB, Lau CC and Brown PH: Comprehensive genomic analysis identifies novel subtypes and targets of triple-negative breast cancer. Clin Cancer Res 21(7): 1688-1698, 2015. PMID: 25208879. DOI: 10.1158/10780432.CCR-14-0432

6 Okabe M, Toh U, Iwakuma N, Saku S, Akashi M, Kimitsuki Y, Seki N, Kawahara A, Ogo E, Itoh K and Akagi Y: Predictive factors of the tumor immunological microenvironment for longterm follow-up in early stage breast cancer. Cancer Sci 108(1): 81-90, 2017. PMID: 27801993. DOI: 10.1111/cas.13114

7 Tomioka N, Azuma M, Ikarashi M, Yamamoto M, Sato M, Watanabe KI, Yamashiro K and Takahashi M: The therapeutic candidate for immune checkpoint inhibitors elucidated by the status of tumor-infiltrating lymphocytes (TILs) and programmed death ligand 1 (PD-L1) expression in triple negative breast cancer (TNBC). Breast Cancer, 2017. PMID: 28488168. DOI: 10.1007/s12282-017-0781-0

8 Stanton SE, Adams S and Disis ML: Variation in the incidence and magnitude of tumor-infiltrating lymphocytes in breast cancer subtypes: A systematic review. JAMA Oncol 2(10): 1354-1360, 2016. PMID: 27355489. DOI: 10.1001/jamaoncol.2016.1061

9 Adams S, Gray RJ, Demaria S, Goldstein L, Perez EA, Shulman LN, Martino S, Wang M, Jones VE, Saphner TJ, Wolff AC, Wood WC, Davidson NE, Sledge GW, Sparano JA and Badve SS: Prognostic value of tumor-infiltrating lymphocytes in triplenegative breast cancers from two phase III randomized adjuvant breast cancer trials: ECOG 2197 and ECOG 1199. J Clin Oncol 32(27): 2959-2966, 2014. PMID: 25071121. DOI: 10.1200/ JCO.2013.55.0491

10 Dieci MV, Mathieu MC, Guarneri V, Conte P, Delaloge S, Andre $\mathrm{F}$ and Goubar A: Prognostic and predictive value of tumorinfiltrating lymphocytes in two phase III randomized adjuvant breast cancer trials. Ann Oncol 26(8): 1698-1704, 2015. PMID: 25995301. DOI: 10.1093/annonc/mdv239

11 Ibrahim EM, Al-Foheidi ME, Al-Mansour MM and Kazkaz GA: The prognostic value of tumor-infiltrating lymphocytes in triplenegative breast cancer: A meta-analysis. Breast Cancer Res Treat
148(3): 467-476, 2014. PMID: 25361613. DOI: 10.1007/s10549014-3185-2

12 Leon-Ferre RA, Polley MY, Liu H, Gilbert JA, Cafourek V, Hillman DW, Elkhanany A, Akinhanmi M, Lilyquist J, Thomas A, Negron V, Boughey JC, Liu MC, Ingle JN, Kalari KR, Couch FJ, Visscher DW and Goetz MP: Impact of histopathology, tumor-infiltrating lymphocytes, and adjuvant chemotherapy on prognosis of triple-negative breast cancer. Breast Cancer Res Treat 167(1): 89-99, 2018. PMID: 28913760. DOI: 10.1007/s10549-017-4499-7

13 Loi S, Michiels S, Salgado R, Sirtaine N, Jose V, Fumagalli D, Kellokumpu-Lehtinen PL, Bono P, Kataja V, Desmedt C, Piccart MJ, Loibl S, Denkert C, Smyth MJ, Joensuu H and Sotiriou C: Tumor-infiltrating lymphocytes are prognostic in triple negative breast cancer and predictive for trastuzumab benefit in early breast cancer: Results from the finher trial. Ann Oncol 25(8): 1544-1550, 2014. PMID: 24608200. DOI: 10.1093/annonc/ mdu112

14 Park IH, Kong SY, Ro JY, Kwon Y, Kang JH, Mo HJ, Jung SY, Lee S, Lee KS, Kang HS, Lee E, Joo J and Ro J: Prognostic implications of tumor-infiltrating lymphocytes in association with programmed death ligand 1 expression in early-stage breast cancer. Clin Breast Cancer 16(1): 51-58, 2016. PMID: 26364145. DOI: 10.1016/j.clbc.2015.07.006

15 Pruneri G, Gray KP, Vingiani A, Viale G, Curigliano G, Criscitiello C, Lang I, Ruhstaller T, Gianni L, Goldhirsch A, Kammler R, Price KN, Cancello G, Munzone E, Gelber RD, Regan MM and Colleoni M: Tumor-infiltrating lymphocytes (TILs) are a powerful prognostic marker in patients with triple-negative breast cancer enrolled in the IBCSG phase III randomized clinical trial 22-00. Breast Cancer Res Treat 158(2): 323-331, 2016. PMID: 27372069. DOI: $10.1007 / \mathrm{s} 10549-016-3863-3$

16 Polonia A, Pinto R, Cameselle-Teijeiro JF, Schmitt FC and Paredes J: Prognostic value of stromal tumour-infiltrating lymphocytes and programmed cell death-ligand 1 expression in breast cancer. J Clin Pathol 70(10): 860-867, 2017. PMID: 28373294. DOI: 10.1136/jclinpath-2016-203990

17 Pruneri G, Vingiani A, Bagnardi V, Rotmensz N, De Rose A, Palazzo A, Colleoni AM, Goldhirsch A and Viale G: Clinical validity of tumor-infiltrating lymphocytes analysis in patients with triple-negative breast cancer. Ann Oncol 27(2): 249-256, 2016. PMID: 26598540. DOI: 10.1093/annonc/mdv571

18 Denkert C, von Minckwitz G, Brase JC, Sinn BV, Gade S, Kronenwett R, Pfitzner BM, Salat C, Loi S, Schmitt WD, Schem C, Fisch K, Darb-Esfahani S, Mehta K, Sotiriou C, Wienert S, Klare P, Andre F, Klauschen F, Blohmer JU, Krappmann K, Schmidt M, Tesch H, Kummel S, Sinn P, Jackisch C, Dietel M, Reimer T, Untch $\mathrm{M}$ and Loibl S: Tumor-infiltrating lymphocytes and response to neoadjuvant chemotherapy with or without carboplatin in human epidermal growth factor receptor 2-positive and triple-negative primary breast cancers. J Clin Oncol 33(9): 983-991, 2015. PMID: 25534375. DOI: 10.1200/JCO. 2014.58.1967

19 Issa-Nummer Y, Darb-Esfahani S, Loibl S, Kunz G, Nekljudova V, Schrader I, Sinn BV, Ulmer HU, Kronenwett R, Just M, Kuhn T, Diebold K, Untch M, Holms F, Blohmer JU, Habeck JO, Dietel M, Overkamp F, Krabisch P, von Minckwitz G and Denkert C: Prospective validation of immunological infiltrate for prediction of response to neoadjuvant chemotherapy in HER2negative breast cancer-a substudy of the neoadjuvant Geparquinto trial. PLoS One 8(12): e79775, 2013. PMID: 24312450. DOI: 10.1371/journal.pone.0079775 
20 Loi S, Sirtaine N, Piette F, Salgado R, Viale G, Van Eenoo F, Rouas G, Francis P, Crown JP, Hitre E, de Azambuja E, Quinaux E, Di Leo A, Michiels S, Piccart MJ and Sotiriou C: Prognostic and predictive value of tumor-infiltrating lymphocytes in a phase III randomized adjuvant breast cancer trial in node-positive breast cancer comparing the addition of docetaxel to doxorubicin with doxorubicin-based chemotherapy: BIG 02-98. J Clin Oncol 31(7): 860-867, 2013. PMID: 23341518. DOI: 10.1200/JCO 2011.41 .0902

21 Salgado R, Denkert C, Demaria S, Sirtaine N, Klauschen F, Pruneri G, Wienert S, Van den Eynden G, Baehner FL, PenaultLlorca F, Perez EA, Thompson EA, Symmans WF, Richardson AL, Brock J, Criscitiello C, Bailey H, Ignatiadis M, Floris G, Sparano J, Kos Z, Nielsen T, Rimm DL, Allison KH, Reis-Filho JS, Loibl S, Sotiriou C, Viale G, Badve S, Adams S, WillardGallo $\mathrm{K}$ and Loi S: The evaluation of tumor-infiltrating lymphocytes (TILs) in breast cancer: Recommendations by an International TILs Working Group 2014. Ann Oncol 26(2): 259271, 2015. PMID: 25214542. DOI: 10.1093/annonc/mdu450

22 Denkert C, Loibl S, Noske A, Roller M, Muller BM, Komor M, Budczies J, Darb-Esfahani S, Kronenwett R, Hanusch C, von Torne C, Weichert W, Engels K, Solbach C, Schrader I, Dietel $\mathrm{M}$ and von Minckwitz G: Tumor-associated lymphocytes as an independent predictor of response to neoadjuvant chemotherapy in breast cancer. J Clin Oncol 28(1): 105-113, 2010. PMID: 19917869. DOI: 10.1200/JCO.2009.23.7370
23 Lakhani SR EI, Schnitt SJ, Tan PH and van de Vijver MJ (eds.): WHO Classification of Tumours of the Breast. Fourth Edition. Lyon: International Agency for Research on Cancer, 2012.

24 Mao Y, Qu Q, Chen X, Huang O, Wu J and Shen K: The prognostic value of tumor-infiltrating lymphocytes in breast cancer: A systematic review and meta-analysis. PLoS One 11(4): e0152500, 2016. PMID: 27073890. DOI: 10.1371/journal.pone. 0152500

25 Mahmoud SM, Paish EC, Powe DG, Macmillan RD, Grainge MJ, Lee AH, Ellis IO and Green AR: Tumor-infiltrating CD8+ lymphocytes predict clinical outcome in breast cancer. J Clin Oncol 29(15): 1949-1955, 2011. PMID: 21483002. DOI: 10.1200/JCO.2010.30.5037
Received April 26, 2019

Revised May 13, 2019

Accepted May 15, 2019 